\title{
Meshless computation for partial differential equations of fractional order
}

\author{
P. H. Wen ${ }^{1} \&$ Y. C. Hon ${ }^{2}$

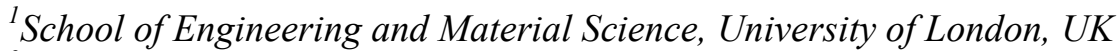 \\ ${ }^{2}$ Department of Mathematics, City University of Hong Kong, \\ Hong Kong SAR, China
}

\begin{abstract}
Fractional advection-dispersion equations are used in groundwater hydrology to model the transport of passive tracers carried by fluid flow in a porous medium. In this paper, the numerical properties of partial differential equations of fractional order $1 \leq \alpha \leq 2$ are investigated by the use of a radial basis function interpolation scheme. The differential equations of fractional order are first analyzed in the Laplace transformed domain and the Durbin inversion method is then used to determine the solutions in the time domain. The accuracy and stability of these methods are investigated for several standard types of problems involving partial differential equations of fractional order.
\end{abstract}

Keywords: fractional derivatives, partial differential equations, radial basis function, Laplace transform, Durbin algorithm.

\section{Introduction}

Analysis of the diffusion-wave equation in mathematical physics has been of considerable interest in the literature. Ordinary and partial differential equations of fractional order have been the focus of many studies because of their frequent appearance in various applications in fluid mechanics, viscoelasticity, biology, physics, and engineering. Fractional calculus in mathematics is a natural extension of integer-order calculus and gives a useful mathematical tool for modeling many processes in nature. One of these processes, in which fractional derivatives have been successfully applied, is called diffusion [1]. Fractional derivatives have recently been applied to many problems in physics [2-8], finance $[9,10]$, and hydrology [11]. Fractional space derivatives are used to 
model anomalous diffusion or dispersion, where a particle plume spreads at a rate inconsistent with the classical Brownian motion model. When a fractional derivative replaces the second derivative in a diffusion or dispersion model, it leads to enhanced diffusion (also called super diffusion). For one dimensional advection-dispersion model with constant coefficients, analytical solutions are available using Fourier transform methods $[12,13]$. Many practical problems, however, are formulated with variable coefficients [14] whose analytical solutions are still not available.

In the last decade, the radial basis functions (RBFs) have been under intensive research in the areas of multivariate function interpolation and partial differential equations solver $[15,16]$. In this paper we propose a numerical scheme by the use of RBFs for solving the ordinary and partial differential equations of fractional order as follow:

Consider a simple form for transport equations

$$
D^{\alpha}(u)+A(x) \frac{\partial u}{\partial t}+B(x) \frac{\partial^{2} u}{\partial t^{2}}+C(x) u=f(x, t), \quad 0<t, 0 \leq x \leq 1
$$

where the coefficients $A(x), B(x)$ and the source team $f(x, t)$ are given. In (1), $D^{\alpha}$ denotes a fractional derivative operator defined as

$$
D^{\alpha}(u)=\frac{1}{\Gamma(m-\alpha)} \frac{\partial^{m}}{\partial x^{m}} \int_{0}^{x} \frac{u(s) d s}{(x-s)^{\alpha-m+1}}
$$

where $\quad \alpha>0$ and $m$ is an integer such that $m>\alpha>m-1$. The boundary conditions and initial conditions are given respectively by

$$
\begin{aligned}
& u(x, 0)=g_{1}(x), \dot{u}(x, 0)=g_{2}(x) \\
& u(0, t)=0 \\
& u_{, x}(0, t)=0
\end{aligned}
$$

or

$$
\begin{aligned}
& u(x, 0)=g_{1}(x), \dot{u}(x, 0)=g_{2}(x) \\
& u(0, t)=0 \\
& u(1, t)=h(t) .
\end{aligned}
$$

For simplicity, we only consider fractional derivatives in space and the index $\alpha$ is assumed to lie within the range $1 \leq \alpha \leq 2$ and therefore $m=2$. By applying the Laplace transformation on equations $(1)$ and $(3,4)$ with consideration of initial condition, we obtain

$$
\begin{aligned}
& D^{\alpha}(\tilde{u})+A(x)\left[p \tilde{u}-g_{1}(x)\right]+B(x)\left[p^{2} \tilde{u}-p g_{1}(x)-g_{2}(x)\right]+C(x) \tilde{u} \\
& =\widetilde{f}(x), 0<t, 0 \leq x \leq 1
\end{aligned}
$$

with the boundary condition 


$$
\widetilde{u}(0)=0, \widetilde{u}_{, x}(0)=0 \text { or } \tilde{u}(0)=0, \tilde{u}(1)=\widetilde{h} .
$$

We note here that although there are several numerical methods developed to solve differential equations of fractional order, there is little information on the numerical solution of partial differential equations of fractional order.

This paper is organized as follows. In Section 2, radial basis function interpolation scheme is introduced. The numerical schemes for the solutions of (1) are then derived in Section 3. In Section 4 several numerical tests and solutions of the transport equilibrium equation are given for the verification of the accuracy and efficiency of the proposed numerical schemes. Finally, conclusion is given in Section 5.

\section{Approximation scheme using radial basis functions}

RBFs have been initially used for scattered data fitting and general multidimensional data interpolation problems, see Ref. [15], and were later applied by Kansa $[17,18]$ for the numerical approximations of various types PDEs. For one dimensional problem, this interpolation becomes very simple. In order to guarantee unique solution of the interpolation problem, a polynomial term should be added to the interpolation. Thus, the interpolated distribution of function $u$ at the point $x$ can be expressed by

$$
u(x)=\sum_{k=1}^{n} R_{k}\left(x, x_{k}\right) a_{k}+\sum_{j=1}^{t} P_{j}(x) b_{j}=\mathbf{R}(x) \mathbf{a}+\mathbf{P}(x) \mathbf{b}
$$

along with the constraints

$$
\sum_{k=1}^{n} P_{j}\left(x_{k}\right) a_{k}=0, \quad 1 \leq j \leq q
$$

where the vector $\mathrm{R}(x)=\left\{R_{1}\left(x, x_{1}\right), R_{2}\left(x, x_{2}\right), \ldots, R_{n}\left(x, x_{n}\right)\right\}$ is the set of radial basis functions at point $x, \mathrm{P}(x)=\left\{P_{1}(x), P_{2}(x), \ldots, P_{q}(x)\right\}$ is the set of polynomial functions, $\left\{x_{k}\right\}_{1}^{n}$ is the set of distributed collocation points in the range $0 \leq x \leq 1$, $P_{j}=x^{j-1}$. Two unknown vectors $\mathrm{a}=\left\{a_{1}, a_{2}, \ldots, a_{n}\right\}^{T}$ and $\mathrm{b}=\left\{b_{1}, b_{2}, \ldots, b_{t}\right\}^{T}$ are to be determined by nodal values respectively. A set of linear equations to determine the coefficients $\mathbf{a}$ and $\mathbf{b}$ can be written, in matrix form, as

$$
\mathbf{R}_{0} \mathbf{a}+\mathbf{P}_{0} \mathbf{b}=\mathbf{u}, \quad \mathbf{P}_{0}^{T} \mathbf{a}=\mathbf{0}
$$

with nodal values $\mathrm{u}=\left\{u_{1}, u_{2}, \ldots, u_{n}\right\}^{T}$ at collocation points $\left\{x_{k}\right\}_{k=1}^{n}$ and matrices 


$$
\begin{gathered}
\mathbf{R}_{0}=\left[\begin{array}{cccc}
R_{1}\left(x_{1}\right) & R_{2}\left(x_{1}\right) & \ldots & R_{n}\left(x_{1}\right) \\
R_{1}\left(x_{2}\right) & R_{2}\left(x_{2}\right) & \ldots & R_{n}\left(x_{2}\right) \\
\cdot & \cdot & \ldots & \cdot \\
\cdot & \cdot & \ldots & \cdot \\
\cdot & \cdot & \ldots & \cdot \\
R_{1}\left(x_{n}\right) & R_{2}\left(x_{n}\right) & \ldots & R_{n}\left(x_{n}\right)
\end{array}\right], \\
\mathbf{P}_{0}=\left[\begin{array}{cccc}
P_{1}\left(x_{1}\right) & P_{2}\left(x_{1}\right) & \ldots & P_{t}\left(x_{1}\right) \\
P_{1}\left(x_{2}\right) & P_{2}\left(x_{2}\right) & \ldots & P_{t}\left(x_{2}\right) \\
\cdot & \cdot & \ldots & \cdot \\
\cdot & \cdot & \ldots & \cdot \\
\cdot & \cdot & \ldots & \cdot \\
P_{1}\left(x_{n}\right) & P_{2}\left(x_{n}\right) & \ldots & P_{t}\left(x_{n}\right)
\end{array}\right] .
\end{gathered}
$$

Solving these equations given in (9) gives

$$
\begin{aligned}
& \mathbf{b}=\left(\mathbf{P}_{0}^{T} \mathbf{R}_{0}^{-1} \mathbf{P}_{0}\right)^{-1} \mathbf{P}_{0}^{T} \mathbf{R}_{0}^{-1} \mathbf{u}=\mathbf{B u}, \\
& \mathbf{a}=\mathbf{R}_{0}^{-1}\left[\mathbf{I}-\mathbf{P}_{0}\left(\mathbf{P}_{0}^{T} \mathbf{R}_{0}^{-1} \mathbf{P}_{0}\right)^{-1} \mathbf{P}_{0}^{T} \mathbf{R}_{0}^{-1}\right] \mathbf{u}=\mathbf{A} \mathbf{u}
\end{aligned}
$$

where I denotes the diagonal unit matrix. In this paper, the radial basis function is selected to be the infinitely smooth multiquadrics:

$$
R_{k}\left(x, x_{k}\right)=\sqrt{c^{2}+\left(x-x_{k}\right)^{2}}
$$

where $c$ is a free parameter. Finally the approximated variable can be presented as

$$
u(x)=[\mathbf{R}(x) \mathbf{A}+\mathbf{P}(x) \mathbf{B}] \mathbf{u}=\sum_{k=1}^{n} N_{k}(x) u_{k}=\mathbf{N u}
$$

where $N_{k}(x)$ is called shape function. From the expression (13), it can be seen that the partial derivatives of the unknown function can be obtained by simply evaluating the partial derivatives of the shape functions. From (13), we have

$$
\begin{aligned}
& u^{\prime}=u_{, x}=\left[\mathbf{R}_{, x}(x) \mathbf{A}+\mathbf{P}_{, x}(x) \mathbf{B}\right] \mathbf{u}=\overline{\mathbf{N}} \mathbf{u} \\
& u^{\prime \prime}=u_{, x x}=\left[\mathbf{R}_{, x x}(x) \mathbf{A}+\mathbf{P}_{, x x}(x) \mathbf{B}\right] \mathbf{u}=\overline{\overline{\mathbf{N}}} \mathbf{u}
\end{aligned}
$$$$
\text { and }
$$

where 


$$
R_{k, x}(x)=\frac{x-x_{k}}{\sqrt{c^{2}+\left(x-x_{k}\right)^{2}}} \text { and } P_{j, x}(x)=(j-1) x^{j-2}
$$

For second order derivative of the shape function, we have

$$
R_{k, x x}(x)=\frac{c^{2}}{\sqrt{c^{2}+\left(x-x_{k}\right)^{2}}} \text { and } P_{j, x x}(x)=(j-1)(j-2) x^{j-3} \text {. }
$$

\section{Numerical scheme of fractional derivatives}

The space variable $x$ is defined to be the unit length interval in (5). By simple integration by part, the fractional derivative can be written as

$$
\int_{0}^{x} \frac{\widetilde{u}(s) d s}{(x-s)^{\alpha-1}}=-\widetilde{u}(0) x^{\alpha}+\frac{\widetilde{u}^{\prime}(0)}{\alpha(\alpha+1)} x^{\alpha+1}+\frac{1}{\alpha(\alpha+1)} \int_{0}^{x}(x-s)^{\alpha+1} \widetilde{u}^{\prime \prime}(s) d s
$$

Therefore, the differential equation with fractional order (5) becomes

$$
\begin{aligned}
& \frac{1}{\Gamma(2-\alpha)} \int_{0}^{x} \frac{\tilde{u}^{\prime \prime}(s) d s}{(x-s)^{\alpha-1}}+p[A(x)+p B(x)] \tilde{u} \\
& +C(x) \tilde{u}+\frac{\tilde{u}(0)}{\Gamma(1-\alpha)} x^{-\alpha}+\frac{\tilde{u}_{, x}(0)}{\Gamma(2-\alpha)} x^{1-\alpha}=G(x) \\
& G(x)=\tilde{f}(x)+A(x) g_{1}(x)+B(x)\left[p g_{1}(x)+g_{2}(x)\right] .
\end{aligned}
$$

It is worthy to notice that there are singularities in (18) at origin if $1 \leq \alpha \leq 2$. It may cause troubles for numerical calculation. Normally the boundary condition $\tilde{u}(0)=\tilde{u}_{, x}(0)=0$ is assumed [19]. Substituting (13) (14) into (18) results

$$
\begin{aligned}
& \frac{1}{\Gamma(2-\alpha)} \int_{0}^{x} \frac{\overline{\overline{\mathbf{N}}}(s) \widetilde{\mathbf{u}} d s}{(x-s)^{\alpha-1}}+\left[p A(x)+p^{2} B(x)+C(x)\right] \mathbf{N}(x) \widetilde{\mathbf{u}} \\
& +\frac{\mathbf{N}(0) \widetilde{\mathbf{u}}}{\Gamma(1-\alpha)} x^{-\alpha}+\frac{\overline{\mathbf{N}}(0) \widetilde{\mathbf{u}}}{\Gamma(2-\alpha)} x^{1-\alpha}=G(x)
\end{aligned}
$$

or 


$$
\left\{\begin{array}{l}
\frac{1}{\Gamma(2-\alpha)} \int_{0}^{x} \frac{\overline{\overline{\mathbf{N}}}(s) d s}{(x-s)^{\alpha-1}}+\left[p A(x)+p^{2} B(x)+C(x)\right] \mathbf{N}(x) \\
+\frac{\mathbf{N}(0)}{\Gamma(1-\alpha)} x^{-\alpha}+\frac{\overline{\mathbf{N}}(0)}{\Gamma(2-\alpha)} x^{1-\alpha}
\end{array}\right\} \widetilde{\mathbf{u}}=G(x)
$$

In this paper, we set up a grid of points, $x_{k}=(k-1) / N, \mathrm{k}=1,2, \ldots, n, n=N+1$. For each collocation point except the end points $x_{1}$ and $x_{N+1}$, we have

$$
\left\{\begin{array}{l}
\frac{1}{\Gamma(2-\alpha)} \int_{0}^{x_{k}} \frac{\overline{\overline{\mathbf{N}}}(s) d s}{\left(x_{k}-s\right)^{\alpha-1}}+\left[p A\left(x_{k}\right)+p^{2} B\left(x_{k}\right)+C\left(x_{k}\right)\right] \mathbf{N}\left(x_{k}\right) \\
+\frac{\mathbf{N}(0)}{\Gamma(1-\alpha)} x_{k}^{-\alpha}+\frac{\overline{\mathbf{N}}(0)}{\Gamma(2-\alpha)} x_{k}^{1-\alpha}
\end{array}\right\} \widetilde{\mathbf{u}}=G\left(x_{k}\right)
$$

for $k=2,3, \ldots, N$, with the boundary conditions

$$
\widetilde{u}(0)=\widetilde{u}_{1}=0, \tilde{u}_{, x}(0)=\sum_{k=1}^{n} \bar{N}_{k}(0) \tilde{u}_{k}=0 \text { or } \tilde{u}(0)=\tilde{u}_{1}=0, \tilde{u}(1)=\tilde{u}_{n}=\tilde{h} .
$$

Thus, we have obtained a complete system of algebraic equations for the computation of $(N+1)$ nodal unknowns $\tilde{\mathrm{u}}$ in the Laplace transform domain. As there is weak singularity in the integral of (10), we can transfer the integral into a regular integral as

$$
\int_{0}^{x_{k}} \frac{F(s) d s}{\left(x_{k}-s\right)^{\alpha-1}}=\frac{1}{2-\alpha} \int_{0}^{x_{k}^{2-\alpha}} F\left(x_{k}-\lambda^{1 /(2-\alpha)}\right) d \lambda
$$

whose integration can be performed by any standard integral scheme.

There are many inversion methods available for numerical computing the inverse Laplace transform. Here, the method proposed by Durbin [20] is adopted. A series of samples $(L+1)$ in the transformation space $p_{l}$, $l=0,1,2, \ldots, L$, are selected as complex variable. Transformed variables are evaluated for these specified transform parameters respectively. The physical variables in the time domain can then be determined by the Laplace inversion technique. Demonstration of the Durbin's inverse method was made by Wen et al $[21,22]$ for the elasticity wave propagations for two and three dimensional problems. The formula of inversion used is written as

$$
F(t)=\frac{2 e^{\omega t}}{T}\left[-\frac{1}{2} \widetilde{F}\left(p_{0}\right)+\sum_{l=0}^{L} \operatorname{Re}\left\{\widetilde{F}\left(p_{l}\right) e^{2 l \pi i t / T}\right\}\right]
$$

where $\tilde{F}\left(p_{l}\right)$ denotes the transformed variable in the Laplace domain and $i=\sqrt{-1}$. The parameter of the Laplace transform is taken to be $s_{l}=\omega+2 l \pi i / T$. The selection of two free parameters $\omega$ and $T$ has small 
effect on the accuracy of inversion. By large number of numerical tests, we can conclude that Durbin inversion method is stable and convergent for large range selection of free parameters $\omega$ and $T$ particularly for the solid dynamic mechanics.

\section{Numerical verification}

In the following test cases, we assume that $1 \leq \alpha \leq 2, m=2$. For numerical error estimation, we define the relative average error by the following formula

$$
\operatorname{MEU}(x)=\frac{1}{N u_{\max }^{*}} \sum_{i=1}^{M}\left|u\left(x, t_{i}\right)-u^{*}\left(x, t_{i}\right)\right|
$$

where $M$ is the number of time observations at collocation point $x$ and $u^{*}$ denotes the analytical solutions at time $t_{i}$ in the region $\left[0, t_{\max }\right]$. The free parameters is chosen to be $\omega=5 / T$ and $T=20$. To test the numerical schemes, it is important to use simple analytical models. Hence, in the first example we consider the case of evolution of a density profile to an equilibrium solution [19]. The equilibrium density profile is the solution of the equation,

$$
D^{\alpha}(u)=f(x), \quad 0 \leq x \leq 1 .
$$

In order to have a simple solution, we use a simple form for the source function

$$
f(x)=1-x^{2-\alpha}
$$

Along with the boundary condition $u(0)=0, u^{\prime}(0)=0$. In this case, the analytical solution for any value of $\alpha>1$ is given by

$$
u^{*}(x)=\frac{x^{\alpha}}{\Gamma(1+\alpha)}-\frac{\Gamma(3-\alpha) x^{2}}{2} .
$$

Numerical results are listed in Table 1 when fractional order $\alpha=1.5$. It is apparent that the accuracy depends on the selection of free parameter $c$. For small $c$, we are able to obtain high accurate results even with small number of collocation points. However, the accuracy does not increase with the increasing of collocation number. In addition, the divergence of solution will occur when $c=10 / n$ and $n>31$ even when double precision of variable is used. This fact is well known in the use of radial basis functions for multivariate interpolation and PDE solver. Therefore, in the following tests, parameter $c$ is chosen to be 
Table 1: $\quad$ Relative error in (23) for the first example

$$
u_{\max }^{*}=1 / \Gamma(1+\alpha)-\Gamma(3-\alpha) / 2 .
$$

\begin{tabular}{|c|c|c|c|c|}
\hline$c$ & $n=11$ & $n=21$ & $n=31$ & $n=41$ \\
\hline $0.1 / n$ & $9.0454 \times 10^{-4}$ & $4.0698 \times 10^{-4}$ & $2.6690 \times 10^{-4}$ & $1.9813 \times 10-4$ \\
\hline 8 & $6.9659 \times 10^{-4}$ & $2.4753 \times 10^{-4}$ & $1.3557 \times 10^{-4}$ & $8.7012 \times 10-5$ \\
\hline $10 / n$ & $1.9478 \times 10^{-4}$ & $1.2055 \times 10^{-4}$ & $4.8050 \times 10^{-1}$ & $5.8850 \times 10-1$ \\
\hline
\end{tabular}

$c=1 / n$. In the second example, we consider the following differential equation with non-zero boundary condition at origin

$$
D^{\alpha}(u)+u(x)+\frac{\partial^{2} u}{\partial x^{2}}=f(x), \quad 0 \leq x \leq 1
$$

along with the boundary condition $u(0)=0, u(1)=1$ and source term

$$
f(x)=\frac{x^{2-\alpha}}{\Gamma(3-\alpha)}+x^{2}+2 .
$$

The analytical solution is $u(x)=x^{2}$. Numerical results are presented in Table 2 for different fractional order $\alpha$.

Table 2: $\quad$ Relative error for the second example, $u_{\max }^{*}=1$.

\begin{tabular}{|c|c|c|c|}
\hline & $n=5$ & $n=11$ & $n=21$ \\
\hline 1.001 & $9.7484 \times 10^{-8}$ & $2.3098 \times 10^{-8}$ & $8.7972 \times 10^{-8}$ \\
\hline 1.1 & $1.8599 \times 10^{-8}$ & $2.1025 \times 10^{-8}$ & $1.7394 \times 10^{-8}$ \\
\hline 1.5 & $6.5070 \times 10^{-9}$ & $1.2149 \times 10^{-8}$ & $3.4291 \times 10^{-9}$ \\
\hline 1.9 & $2.2595 \times 10^{-8}$ & $2.4772 \times 10^{-8}$ & $9.4626 \times 10^{-7}$ \\
\hline 1.999 & $1.6621 \times 10^{-7}$ & $2.2757 \times 10^{-6}$ & $3.4531 \times 10^{-7}$ \\
\hline
\end{tabular}

For different fractional order, same order of accuracy can be achieved even with different number of collocation points. Apparently this numerical scheme is of high accuracy and convergent to solve partial differential equation of fractional order. In the final example, we consider the following differential equation of fractional order with time dependence as 


$$
\begin{gathered}
D^{\alpha}(u)-e^{x}\left(\frac{\partial u}{\partial t}+\frac{\partial^{2} u}{\partial t^{2}}\right)=f(x, t), \\
f(x, t)=\left(t+e^{x}\right) e^{-t} / \Gamma(1+\alpha), 0<t, 0 \leq x \leq 1 \\
u(x, 0)=0, \frac{\partial u(x, 0)}{\partial t}=x^{\alpha} \\
u(0, t)=0, u^{\prime}(0, t)=0 .
\end{gathered}
$$

The analytical solution is determined by $u^{*}(x, t)=x^{\alpha} t e^{-t}$. The numerical solutions by using the proposed scheme are plotted in Figure 1 for $0 \leq t \leq 5$. In this case fractional order is fixed to be $\alpha=1.5$. Two curves are plotted which correspond to the numbers of collocation point $n=21$ and 41 respectively.

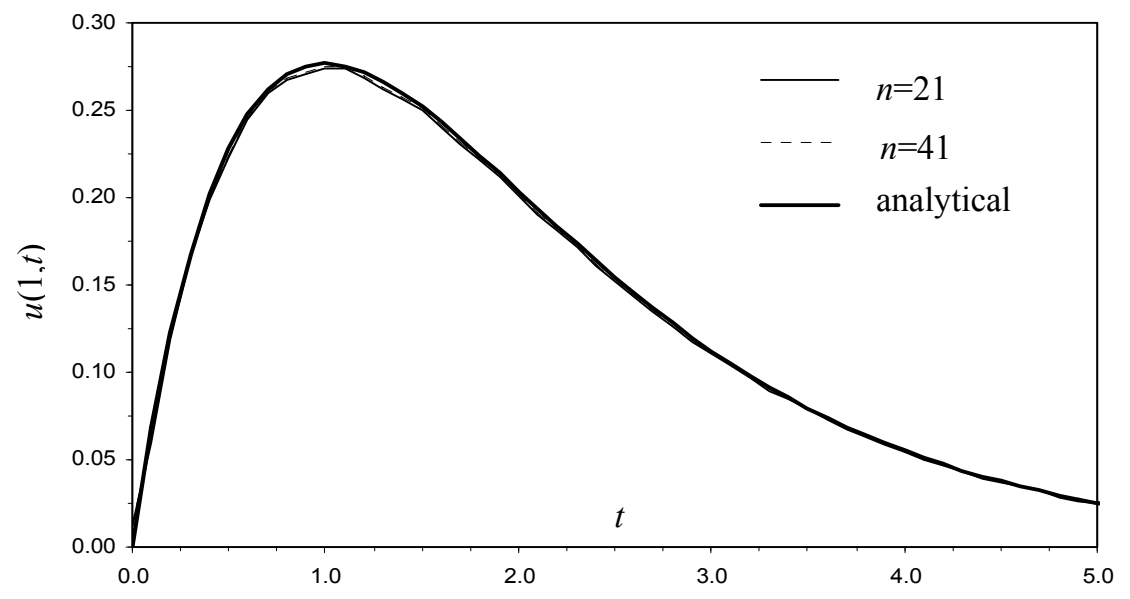

Figure 1: Variation of $u(1, t)$ against time $t$.

\section{Conclusion}

In this paper we consider a partial differential equation of fractional order $\alpha$ with variable coefficient and propose a new numerical scheme using radial basis function interpolation. For zero boundary conditions, $u(0, t)=u^{\prime}(0, t)=0$, the singularities are vanished in the differential equation and accurate numerical solutions can be obtained. Although this is not required, for simplicity, all collocation points are chosen to be uniformly distributed in the range $0 \leq x \leq 1$. The Laplace transform technique with Durbin inversion method for time variable is applied for solving the time dependent problems. Compared with analytical 
solutions, excellent agreement with the proposed numerical scheme is obtained. We can conclude with following observations: (1) The radial basis function interpolation is suitable to solve partial differential equation of fractional order; (2) The time dependent problem can be dealt with in the Laplace transform domain; (3) Numerical solutions are not sensitive with the selections of collocation point number $n$; (4) Nonlinear problems will be investigated in separate paper.

\section{Acknowledgement}

The work described in this paper was fully supported by a grant from the Research Grants Council of the Hong Kong Special Administrative Region, China (Project No. CityU 101310).

\section{References}

[1] Ciesielski M. and Leszczynsky J., Numerical treatment of an initialboundary value problem for fractional partial differential equations, Signal Process 86 (2006), 2619-2631.

[2] Barkai E., Metzler R., Klafter J., From continuous time random walks to the fractional Fokker-Planck equation, Phys. Rev. E 61 (2000) 132-138.

[3] Blumen A., Zumofen G., Klafter J., Transport aspects in anomalous diffusion: Levy walks, Phys. Rev. A 40 (1989) 3964-3973.

[4] Bouchaud J. P., Georges A., Anomalous diffusion in disordered mediastatistical mechanisms, models and physical applications, Phys. Rep. 195 (1990) 127-293.

[5] Chaves A., Fractional diffusion equation to describe Levy lights, Phys. Lett. A 239 (1998) 13-16.

[6] Meerschaert M. M., Benson D., ScheXer H. P., Baeumer B., Stochastic solution of space-time fractional diffusion equations, Phys. Rev. E 65 (2002) 1103-1106.

[7] Meerschaert M.M., ScheXer H. P., Semistable Levy Motion, Frac. Calc. Appl. Anal. 5 (2002) 27-54.

[8] Zaslavsky G., Fractional kinetic equation for Hamiltonian chaos. Chaotic advection, tracer dynamics and turbulent dispersion, Phys. D 76 (1994) $110-122$.

[9] Raberto M., Scalas E., Mainardi F., Waiting-times and returns in highfrequency financial data: an empirical study, Physica A 314 (2002) 749755.

[10] Sabatelli L., Keating S., Dudley J., Richmond P., Waiting time distributions in financial markets, Eur. Phys. J. B 27 (2002) 273-275.

[11] Baeumer B., Meerschaert M. M., Benson D., Wheatcraft S. W., Subordinated advection-dispersion equation for contaminant transport, Water Resour. Res. 37 (2001) 1543-1550.

[12] Benson D., Wheatcraft S., Meerschaert M. M., Application of a fractional advection-dispersion equation, Water Resour. Res. 36 (2000) 1403-1412. 
[13] Chaves A., Fractional diffusion equation to describe Levy lights, Phys. Lett. A 239 (1998) 13-16.

[14] Barkai E., Metzler R., Klafter J., From continuous time random walks to the fractional Fokker-Planck equation, Phys. Rev. E 61 (2000) 132-138.

[15] Hardy R. L., Multiquadric equations of topography and other irregular surfaces, J. Geophys. Res. 176, (1971) 1905-1915.

[16] Franke, R., Scattered data interpolation: test of some methods, Math. Comput. 38, (1982) 181-200.

[17] Kansa, E. J., Multiquadrics - a scattered data approximation scheme with applications to computational fluid dynamics-I, Computers Math. Appl. 19(8/9), (1990) 127-145.

[18] Madych, W. R., and Nelson, S. A., Multivariate interpolation and conditionally positive definite functions I, J. Approx. Theory and Its Appl. 4, (1988) 77-89.

[19] Lynch V. E., Carreras B. A., del-Castillo-Negrete D., Ferreira-Mejias K. M., Hicks H. R., Numerical methods for the solution of partial differential equations of fractional order, Journal of Computational Physics 192 (2003) 406-421.

[20] Durbin F., Numerical inversion of Laplace transforms: an efficient improvement to Dubner and Abate's method. The Computer Journal 1975; 17:371-376.

[21] Wen P. H., Chen C. S., The method of particular solutions for solving scalar wave equations. Commun. Numer. Meth. Engng. (2009).

[22] Wen P. H., Aliabadi M. H., Rooke D. P., The influence of elastic waves on dynamic stress intensity factors (three dimensional problem). Archive of Applied Mechanics 1996; 66(6):384-385. 